\title{
Possible valence-bond condensation in the frustrated cluster
}

\author{
magnet $\mathrm{LiZn}_{2} \mathrm{Mo}_{3} \mathrm{O}_{8}$
}

\author{
J.P. Sheckelton, J.R. Neilson, D.G. Soltan, and T.M. McQueen
}

Department of Chemistry, Department of Physics and Astronomy, and the Institute for Quantum Matter, The Johns Hopkins University, Baltimore, MD 21218

The emergence of complex electronic behaviour from simple ingredients has resulted in the discovery of numerous states of matter. Many examples are found in systems exhibiting geometric magnetic frustration, which prevents simultaneous satisfaction of all magnetic interactions. This frustration gives rise to complex magnetic properties such as chiral spin structures ${ }^{1,2,3}$ orbitally-driven magnetism ${ }^{4}$, spin-ice behavior ${ }^{5}$ exhibiting Dirac strings with magnetic monopoles ${ }^{6}$, valence bond solids $^{7,8}$, and spin liquids ${ }^{9,10}$. Here we report the synthesis and characterization of $\operatorname{LiZn}_{2} \mathrm{Mo}_{3} \mathrm{O}_{8}$, a geometrically frustrated antiferromagnet in which the magnetic moments are localized on small transition metal clusters rather than individual ions ${ }^{11,12,13}$. By doing so, first order Jahn-Teller instabilities and orbital ordering are prevented, allowing the strongly interacting magnetic clusters in $\mathrm{LiZn}_{2} \mathrm{Mo}_{3} \mathrm{O}_{8}$ to probably give rise to an exotic condensed valence-bond ground state reminiscent of the proposed resonating valence bond state ${ }^{14,15}$. Our results also link magnetism on clusters to geometric magnetic frustration in extended 
solids, demonstrating a new approach for unparalleled chemical control and tunability in the search for collective, emergent electronic states of matter ${ }^{16,17}$.

Numerous materials possess a geometrically frustrated arrangement of magnetic atoms; such materials have their magnetic moments arranged on frustrated topologies such as triangular lattices $^{18,19}$, kagomé lattices ${ }^{20,21}$, hyper-kagomé lattices ${ }^{22}$, and edge sharing tetrahedra ${ }^{23,24}$. A rich diversity of properties result depending on the magnitude of the per-site spin and orbital occupancies. Yet, the presence of local structural distortions ${ }^{25,26}$, and a degree of site mixing between non-magnetic and magnetic layers ${ }^{27}$ are still key limiters in the quest for new quantum states of matter ${ }^{28}$. Here we show that these problems can be overcome through the use of clusters in which a magnetic, unpaired electron is delocalized over a small number of transition metal atoms, rather than individual magnetic ions, by demonstrating that the $S=1 / 2$ cluster magnet $\mathrm{LiZn}_{2} \mathrm{Mo}_{3} \mathrm{O}_{8}$ is geometrically frustrated and likely possesses a condensed valence bond ground state.

$\mathrm{LiZn}_{2} \mathrm{Mo}_{3} \mathrm{O}_{8}$ is built of discrete $\mathrm{Mo}_{3} \mathrm{O}_{13}$ cluster units (figure 1a), in which all Mo atoms are on equivalent crystallographic sites ${ }^{29}$. The average formal oxidation state of Mo is +3.67 . Each cluster has seven valence electrons. By a simple electron count, supported by molecular orbital calculations (figure 1b), six of these electrons localize into Mo-Mo bonds holding the cluster together. The seventh electron remains unpaired in a totally symmetric (A1 irreducible representation) molecular orbital with equal contributions from all three Mo atoms. The result is one $S=1 / 2$ magnetic moment on each $\mathrm{Mo}_{3} \mathrm{O}_{13}$ cluster. This cluster can then replace an atom as the basic building block of a geometrically frustrated magnetic system, when appropriately arranged. There have been previous reports of systems built of magnetic clusters ${ }^{30}$. In those cases, the unpaired, magnetic electrons within a cluster are still (just like the non-cluster cases) localized on 
individual atoms. By contrast, in $\mathrm{LiZn}_{2} \mathrm{Mo}_{3} \mathrm{O}_{8}$, the magnetism arises from a collective contribution of all three atoms in the $\mathrm{Mo}_{3} \mathrm{O}_{13}$ cluster. This gives rise to a $\mathrm{S}=1 / 2$ moment delocalized over three Mo atoms. This delocalized nature of the moment contributes to the stability of the system and renders the structure impervious to first order Jahn-Teller distortions.

These $\mathrm{Mo}_{3} \mathrm{O}_{13}$ clusters in $\mathrm{LiZn}_{2} \mathrm{Mo}_{3} \mathrm{O}_{8}$ connect at corners, making a two-dimensional $\mathrm{Mo}_{3} \mathrm{O}_{8}$ layer (figure 1c). The $2.6 \AA$ Mo-Mo distance within each cluster is substantially shorter than between clusters $(3.2 \AA)$ reflecting strong metal-metal bonding within each cluster. These $\mathrm{Mo}_{3} \mathrm{O}_{8}$ layers are separated by non-magnetic $\mathrm{Li} / \mathrm{Zn}$ ions (figure $1 \mathrm{~d}$ ) to form the full structure with $R \overline{3} m$ symmetry. Consequently, $\mathrm{LiZn}_{2} \mathrm{Mo}_{3} \mathrm{O}_{8}$ contains two-dimensional layers in which $S=$ $1 / 2 \mathrm{Mo}_{3} \mathrm{O}_{13}$ clusters are arranged on the geometrically frustrated triangular lattice.

The temperature dependence of the magnetic susceptibility of $\mathrm{LiZn}_{2} \mathrm{Mo}_{3} \mathrm{O}_{8}$ shows unusual and unexpected behavior. At temperatures above $96 \mathrm{~K}$, the inverse magnetic susceptibility (figure 2a) is well described by the Curie-Weiss law for paramagnetic spins. A Weiss temperature of $\theta=-220 \mathrm{~K}$ indicates a net mean-field antiferromagnetic interaction between unpaired spins on $\mathrm{Mo}_{3} \mathrm{O}_{13}$ clusters. A Curie constant of $C=0.24 \mathrm{emu} \cdot \mathrm{K} \cdot \mathrm{Oe}^{-1} \cdot \mathrm{mol} f \cdot u^{-1}$ ( $p_{\text {eff }}=1.39$ ) is reduced from the ideal 0.375 value for a free $S=1 / 2$ moment. This may be due to a number of possibilities (see SI), but the most likely is a partial unquenched orbital contribution to the moment, due to spin-orbit coupling. It is not due to the formation of a metallic state: resistivity data (figure S6) shows that $\mathrm{LiZn}_{2} \mathrm{Mo}_{3} \mathrm{O}_{8}$ is electrically insulating at all accessible temperatures. Furthermore, the molecular calculations predict an on-site (cluster) Hubbard $U$ of $\sim 1.2 \mathrm{eV}$, which, depending on bandwidth, could open a gap and explain the insulating behavior. Together, these data imply that the one unpaired electron per cluster in $\mathrm{LiZn}_{2} \mathrm{Mo}_{3} \mathrm{O}_{8}$ behaves as a localized effective $S=1 / 2$ magnetic system (with a partial unquenched orbital contribution just 
like $\mathrm{Co}^{2+}$ or $\mathrm{Cu}^{2+}$ ), and that the magnetic interactions between clusters are strong and antiferromagnetic.

A change in the slope of the inverse magnetic susceptibility as a function of temperature occurs around $T=96 \mathrm{~K}$, with a second linear region of $\chi(T)$ present below this transition (or crossover). A fit to the linear region from $T=2 \mathrm{~K}$ to $T=96 \mathrm{~K}$ gives a Weiss temperature of $\theta=-$ $14 \mathrm{~K}$ and a Curie constant of $C=0.08$. This Curie constant is one-third that of the high temperature value, indicating that two-thirds of the spins contribute negligibly to the magnetic susceptibility below $T=96 \mathrm{~K}$ as the Curie constant scales with the number of moments.

Neutron powder diffraction experiments at $T=12 \mathrm{~K}$ indicate that long-range magnetic order does not develop below the $T \approx 96 \mathrm{~K}$ transition (figure $\mathrm{S} 1$ ). Instead, our results are consistent with two-thirds of the effective spins condensing into magnetic singlets. Although our data are not sufficient to unambiguously determine whether these singlets are static, making a valence-bond solid, or dynamic, making a resonating valence-bond state, neutron powder diffraction data suggest that the singlets are indeed dynamic: at $T=12 \mathrm{~K}$, $\mathrm{LiZn}_{2} \mathrm{Mo}_{3} \mathrm{O}_{8}$ maintains the trigonal $R \overline{3} m$ symmetry that exists at $T=300 \mathrm{~K}$. In most cases, static singlets form a valence bond network and distort the lattice to a lower symmetry. Unambiguous determination of the ground state warrants further study, but the ground state of $\mathrm{LiZn}_{2} \mathrm{Mo}_{3} \mathrm{O}_{8}$ is unusual and reflective of the strong geometric magnetic frustration.

Changes in the experimentally measured heat capacity further elucidate the unusual electronic behavior in $\mathrm{LiZn}_{2} \mathrm{Mo}_{3} \mathrm{O}_{8}$ (figure 2b). $\mathrm{LiZn}_{2} \mathrm{Mo}_{3} \mathrm{O}_{8}$ does not undergo a transition to long range magnetic order above $T=0.1 \mathrm{~K}$ : there is no sharp lambda transition of the heat capacity as a function of temperature. Instead there is only an upturn in the specific heat capacity data below $T=1 \mathrm{~K}$. Applied magnetic fields of $\mu_{o} H=1 \mathrm{~T}$ and $\mu_{o} H=9 \mathrm{~T}$ (figure $2 \mathrm{~b}$ inset) 
radically modulate the behavior of the low temperature data. Such large changes from small magnetic fields are surprising given the large Weiss temperature and are likely a result of magnetic frustration in the system. Geometric frustration prevents the formation of long range order and results in low-lying magnetic excitations perturbed by an applied field. Simple models, such as a multilevel Schottky anomaly, do not adequately describe the low temperature data (see SI); further studies are needed to examine and understand the behavior in detail.

The magnetic entropy change of $\mathrm{LiZn}_{2} \mathrm{Mo}_{3} \mathrm{O}_{8}$, accounting for the extra lattice contribution from lithium compared to $\mathrm{Zn}_{2} \mathrm{Mo}_{3} \mathrm{O}_{8}$ (figure 2c, figure S2) also indicates the condensation of two-thirds of the available spins. The total expected magnetic entropy change for $\mathrm{S}=1 / 2$ system is $R \cdot \ln (2)\left(=5.76 \mathrm{~J} \cdot \mathrm{K}^{-1} \cdot \mathrm{mol} f \cdot u^{-1}\right)$, compared to the experimental value of $8(3)$ $\mathrm{J} \cdot \mathrm{K}^{-1} \cdot \mathrm{mol} f \cdot u^{-1}$ from $T=0.1$ to $T=400 \mathrm{~K}$. On cooling from $T=400 \mathrm{~K}$, we observe a gradual and continuous loss of entropy, approximately two-thirds of the expected $\mathrm{S}=1 / 2$ value from $T=400 \mathrm{~K}$ to $T=100 \mathrm{~K}$. Critically, the change in the linear regions of magnetic susceptibility is not accompanied by a sharp transition in the entropy, supporting the claim that these spins condense into singlets, rather than adopt long range magnetic order. Furthermore, the difference in entropy between $T=0.1 \mathrm{~K}$ and $T=100 \mathrm{~K}$ is approximately $\frac{1}{3} R \cdot \ln (2)$, consistent with freezing out of the remaining one-third of spins that did not condense into singlets at $T=96 \mathrm{~K}$.

The resulting magnetic phase diagram of $\mathrm{LiZn}_{2} \mathrm{Mo}_{3} \mathrm{O}_{8}$ is shown in figure $2 \mathrm{~d}$. Near room temperature, the system is paramagnetic and the spins thermally randomize. Cooling below the condensation temperature ( $T \sim 96 \mathrm{~K})$, two-thirds of the spins form a condensed valence bond state. The remaining one-third spins are still paramagnetic and interacting antiferromagnetically until lower temperatures, at which point they lose entropy in a yet-to-be determined manner. 
These results suggest that $\mathrm{LiZn}_{2} \mathrm{Mo}_{3} \mathrm{O}_{8}$ exhibits geometric magnetic frustration between $S$ $=1 / 2$ magnetic clusters and two-thirds of the spins condense into singlets below approximately $T$ $=96 \mathrm{~K}$. Therefore $\mathrm{LiZn}_{2} \mathrm{Mo}_{3} \mathrm{O}_{8}$ is a candidate for a resonating valence-bond state, as there is no evidence for static singlets. More generally, our results show how an extended lattice of magnetic clusters, in place of magnetic ions, produces exotic physics while providing numerous advantages in the design and control of magnetically frustrated materials. This approach opens a new chemical frontier in the search for emergent phenomena in frustrated systems.

\section{Acknowledgements}

This research is supported by the U.S. Department of Energy, Office of Basic Energy Sciences, Division of Materials Sciences and Engineering under Award DE-FG02-08ER46544. Use of the Spallation Neutron Source was supported by the Division of Scientific User Facilities, Office of Basic Energy Sciences, US Department of Energy, under contract DE-AC0500OR22725 with UT-Battelle, LLC. J.P.S. acknowledges the assistance of J. Hodges in collecting and analyzing powder neutron data from POWGEN/SNS. TMM acknowledges useful discussions with O. Tchernyshyov and C. Broholm. 


\section{Methods}

Phase-pure $\mathrm{LiZn}_{2} \mathrm{Mo}_{3} \mathrm{O}_{8}$ was synthesized from a mixture of $\mathrm{Mo}, \mathrm{ZnO}, \mathrm{Li}_{2} \mathrm{MoO}_{4}$, and $\mathrm{MoO}_{2}\left(99+\%\right.$ purity) with an overall starting formula of $\mathrm{LiZn}_{2} \mathrm{Mo}_{3} \mathrm{O}_{8}\left(\mathrm{Li}_{2} \mathrm{Zn}_{2} \mathrm{O}_{3}\right)_{0.2}$. Mo was used as received. $\mathrm{ZnO}$ and $\mathrm{Li}_{2} \mathrm{MoO}_{4}$ were dried at $T=160{ }^{\circ} \mathrm{C}$ overnight. $\mathrm{MoO}_{2}$ was purified by heating overnight under flowing $5 \% \mathrm{H}_{2} / 95 \%$ Ar. The mixtures were pressed into pellets, placed in alumina crucibles, and double-sealed in evacuated, fused silica tubes. The reaction vessel was heated to $T=600^{\circ} \mathrm{C}$ for 24 hours, ramped to $T=1000^{\circ} \mathrm{C}$ at $10^{\circ} \mathrm{C} / \mathrm{hr}$, held for 12 hours, followed by a water quench. The sample was reground and heated again in the same manner. $\mathrm{Zn}_{2} \mathrm{Mo}_{3} \mathrm{O}_{8}$ was synthesized in a similar manner, but with $3 \%$ excess $\mathrm{ZnO}$, and a final temperature of $T=$ $1050{ }^{\circ} \mathrm{C}$.

Magnetization measurements, heat capacities, and resistivities were measured on a sintered pellet in a Quantum Design Physical Properties Measurement System (PPMS) using a dilution refrigerator for $T<2 \mathrm{~K}$ measurements. Heat capacities were measured three times at each temperature using the semi-adiabatic pulse technique, waiting for three time constants per measurement. Data were collected from $T=0.05 \mathrm{~K}$ to $T=400 \mathrm{~K}$ under magnetic fields of $\mu_{o} H=$ $0 \mathrm{~T}, \mu_{o} H=1 \mathrm{~T}$ and $\mu_{o} H=9 \mathrm{~T}$. Magnetic susceptibilities were measured from $T=1.8 \mathrm{~K}$ to $T=$ $320 \mathrm{~K}$ under a $\mu_{o} H=1 \mathrm{~T}$ field. Laboratory X-ray powder diffraction patterns were collected using $\mathrm{Cu}$ K $\alpha$ radiation (1.5418 ̊) on a Bruker D8 Focus diffractometer with a LynxEye detector. Powder neutron diffraction data sets at $T=12 \mathrm{~K}$ with $d$-spacing of $0.2760 \AA \leq d \leq 3.0906 \AA$ and $1.6557 \AA \leq d \leq 8.2415 \AA$ were collected at the Spallation Neutron Source POWGEN diffractometer (BL-11A) at the Oak Ridge National Laboratory and analyzed with the Rietveld method using GSAS with EXPGUI ${ }^{31,32}$. Molecular orbital calculations using density functional theory with the PBE0 functional at the UHF level of theory were performed with the GAMESS software package ${ }^{33}$. 


\section{Author Contributions}

TMM supervised the project. JPS and DES prepared samples. JPS measured neutron diffraction patterns. JPS and TMM measured properties, and JPS, JRN, and TMM analyzed data and prepared the manuscript. 
a

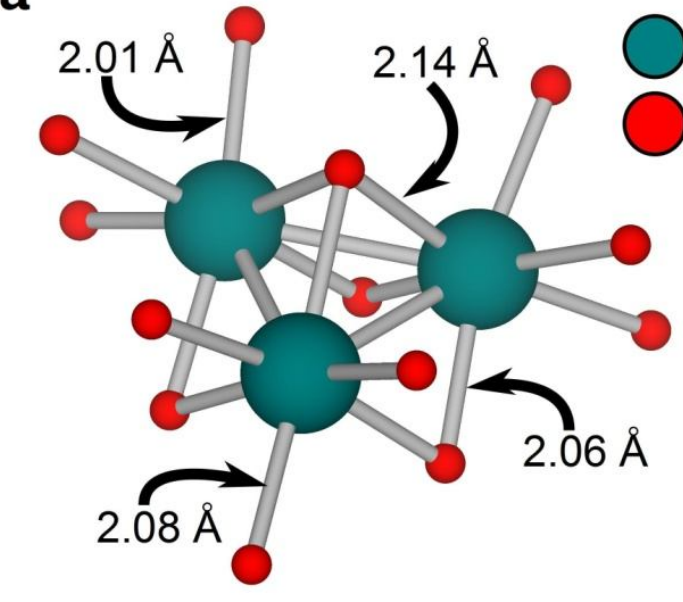

C

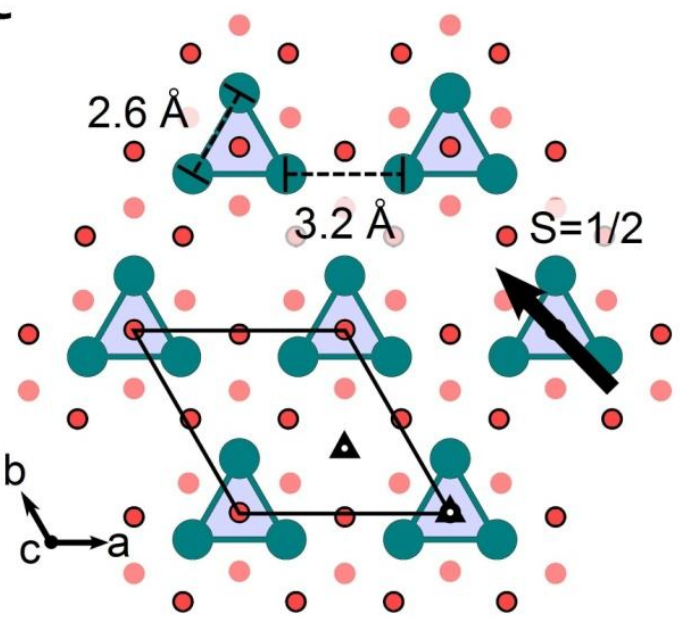

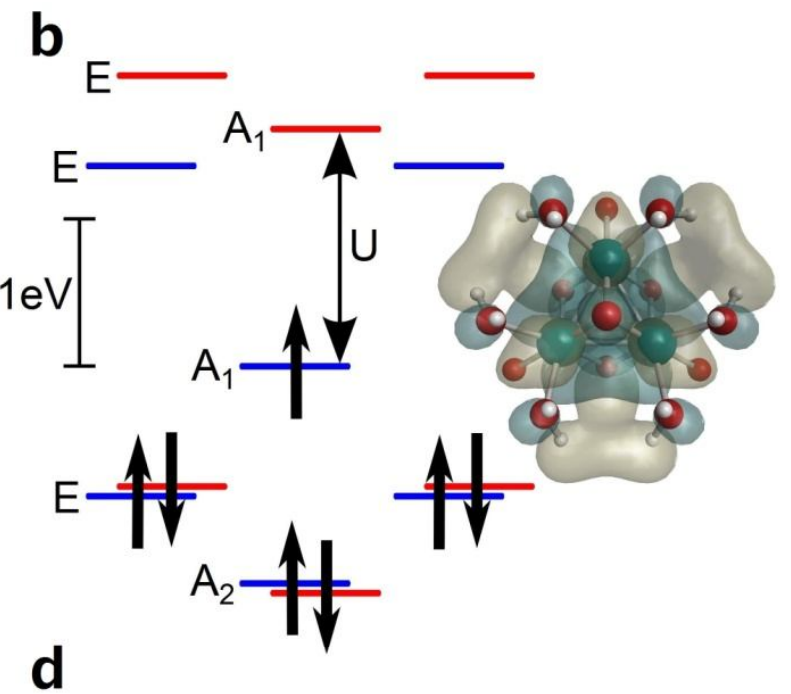

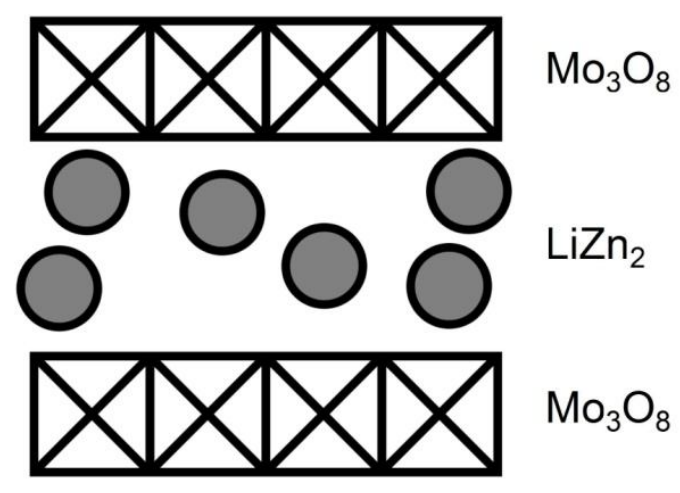

Figure $1 \mid \mathbf{L i Z n}_{2} \mathrm{Mo}_{3} \mathrm{O}_{8}$ structure. a, A single $\mathrm{Mo}_{3} \mathrm{O}_{13}$ cluster shows the local coordination of each Mo atom. b, A spin polarized molecular orbital diagram for $\mathrm{Mo}_{3} \mathrm{O}_{13} \mathrm{H}_{15}\left(C_{3 v}\right)$. There is one unpaired electron per cluster, distributed over all Mo atoms, with a large energy gap to the next available state. The hybrid functional produces an estimate of the on-site repulsion energy, $\mathrm{U} \sim$ $1.2 \mathrm{eV} . \mathrm{A}_{1}, \mathrm{~A}_{2}$ and $\mathrm{E}$ are the irreducible representation labels for each orbital level from the $\mathrm{C}_{3 \mathrm{v}}$ point group. c, Top-down view of the $\mathrm{Mo}_{3} \mathrm{O}_{8}$ layer showing the triangular network formed by the $\mathrm{Mo}_{3} \mathrm{O}_{13} S=1 / 2$ clusters. $\mathbf{d}$, A schematic representation of the magnetic $\mathrm{Mo}_{3} \mathrm{O}_{8}$ layers separated by $\mathrm{LiZn}_{2}$ in $\mathrm{LiZn}_{2} \mathrm{Mo}_{3} \mathrm{O}_{8}$. 

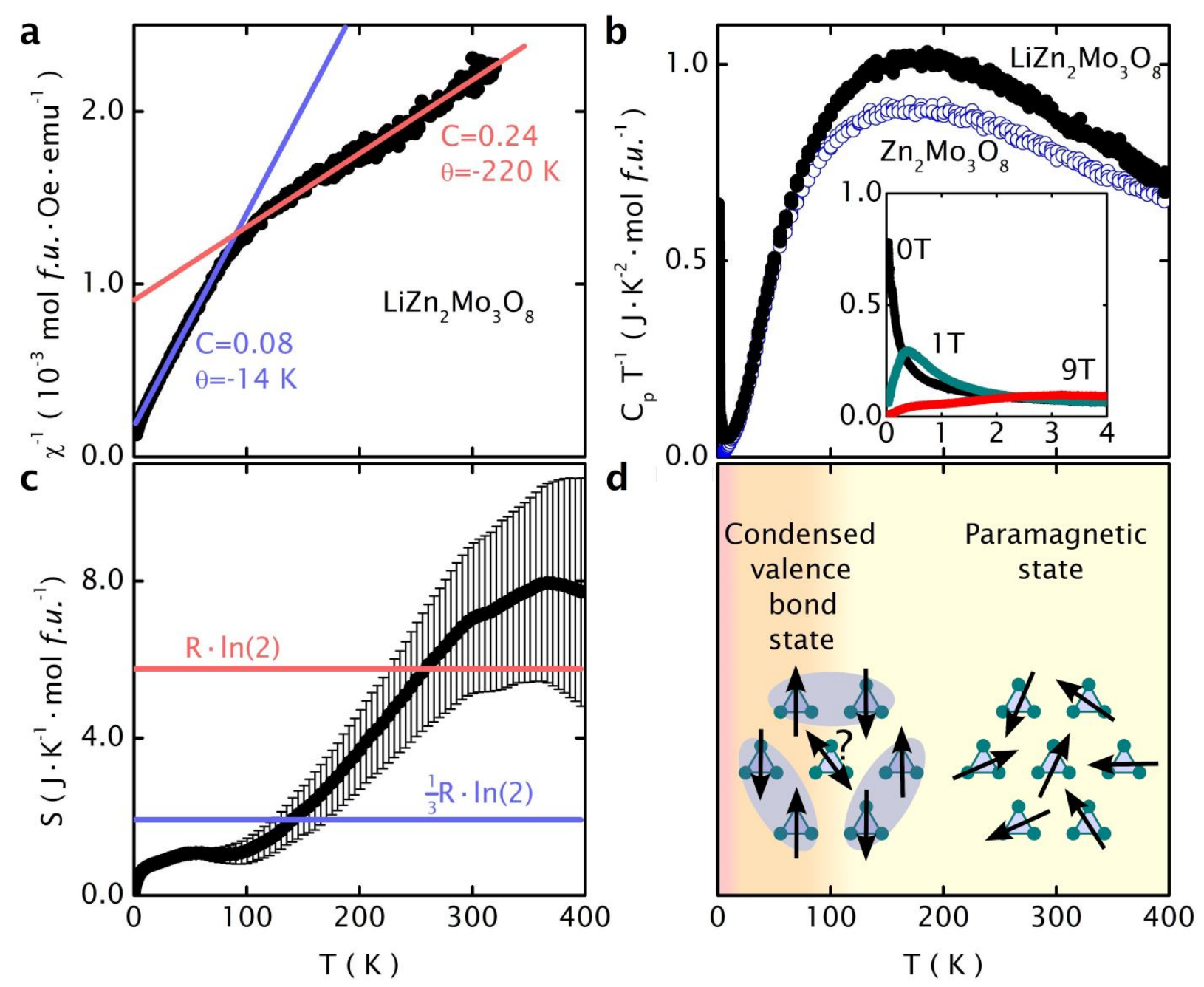

Figure $2 \mid$ Physical properties of $\mathrm{LiZn}_{2} \mathrm{Mo}_{3} \mathrm{O}_{8}$. a, Inverse magnetic susceptibility as a function of temperature for $\mathrm{LiZn}_{2} \mathrm{Mo}_{3} \mathrm{O}_{8}$. Curie-Weiss fits to the two distinct linear portions are shown. Two-thirds of the spins 'disappear' upon cooling below $T=96 \mathrm{~K}$. The Curie constant $\mathrm{C}$ is in units of $\mathrm{emu} \cdot \mathrm{K} \cdot \mathrm{Oe}^{-1} \cdot \operatorname{mol} f \cdot u^{-1} \cdot \mathbf{b}$, Heat capacity divided by temperature as a function of temperature. The inset shows a strong magnetic field dependence of the low temperature specific heat. Data for non-magnetic $\mathrm{Zn}_{2} \mathrm{Mo}_{3} \mathrm{O}_{8}$ is shown for comparison. c, Integrated entropy as a function of temperature. The lattice contribution was subtracted prior to integrating (see SI). Error bars are calculated using standard analysis of error techniques for the propagation of the uncertainty in each $\mathrm{C}_{\mathrm{p}}$ measurement through the numerical integration. This is given by 
$\delta S_{N}=\sum_{i=1}^{N} \frac{x_{i+1}-x_{i}}{2} \sqrt{\left(\delta y_{i+1}\right)^{2}+\left(\delta y_{i}\right)^{2}}$, where the error bars are given by $\delta S_{N}$, and $\delta y_{i}$ is the uncertainty in the $\mathrm{C}_{\mathrm{p}} / \mathrm{T}$ value of the $i^{\text {th }}$ point. $\mathbf{d}$, Proposed magnetic phase diagram of $\mathrm{LiZn}_{2} \mathrm{Mo}_{3} \mathrm{O}_{8}$. Below $T=96 \mathrm{~K}$ the spins enter a condensed valence bond state. 


\section{References}

(1) Bode, M. et al. Chiral magnetic order at surfaces driven by inversion asymmetry. Nature. 447, 190-193. (2007).

(2) Grohol, D. et al. Spin chirality on a two-dimensional frustrated lattice. Nature Materials. 4, 323-328. (2005).

(3) Taguci, Y., Oohara, Y., Yoshizawa, H., Nagaosa, N., Tokura, Y. Spin chirality, berry phase, and anomalous Hall effect in a frustrated ferromagnet. Science. 291, 2573-2576. (2001).

(4) Chen, G. and Balents, L. Spin-orbit coupling in $d^{2}$ ordered double perovskites. Phys. Rev. B. 84, 094420. (2011).

(5) Fennell, T. et al. Magnetic coulomb phase in the spin ice $\mathrm{Ho}_{2} \mathrm{Ti}_{2} \mathrm{O}_{7}$. Science. 326, 415-417. (2009).

(6) Morris, D. J. P. et al. Dirac strings and magnetic monopoles in the spin ice $\mathrm{Dy}_{2} \mathrm{Ti}_{2} \mathrm{O}_{7}$. Science. 326, 411-414. (2009).

(7) Affleck, I., Kennedy, T., Lieb, E. H., Tasaki, H. Rigorous results on valence bond ground states in antiferromagnets. Phys. Rev. Lett. 59, 799-802. (1987)

(8) Matan, K. et al. Pinwheel valence-bond solid and triplet excitations in the two-dimensional deformed kagome lattice. Nature Physics. 6, 865-869. (2010).

(9) Pratt, F. L. et al. Magnetic and non-magnetic phases of a quantum spin liquid. Nature. 471, 612-616. (2011).

(10) Anderson, P. W. The resonating valence bond state in $\mathrm{La}_{2} \mathrm{CuO}_{4}$ and superconductivity. Science. 235, 1196-1198. (1987).

(11) Bramwell, S. T. and Gingras, M. J. P. Spin ice state in frustrated magnetic pyrochlore materials. Science. 294, 1495-1501. (2001).

(12) Carlo, J. P. et al. Triplet and in-gap magnetic states in the ground state of the quantum frustrated fcc antiferromagnet $\mathrm{Ba}_{2} \mathrm{YMoO}_{6}$. Phys. Rev. B. 84, 100404. (2011).

(13) Winterlink, J. et al. Exotic magnetism in the alkali sesquioxides $\mathrm{Rb}_{4} \mathrm{O}_{6}$ and $\mathrm{Cs}_{4} \mathrm{O}_{6}$. Phys. Rev. B. 79, 214410. (2009). 
(14) Anderson, P.W. Resonating valence bonds: a new kind of insulator? Mat. Res. Bull. 8, 153160. (1973).

(15) Anderson, P.W. On the ground state properties of the anisotropic triangular antiferromagnet. Philosophical Magazine. 30, 423-440. (1973).

(16) McCarley, R. E., Metal clusters and extended metal-metal bonding in metal oxide systems. Inorganic Chemistry: Toward the $21^{\text {st }}$ Century. 211, 273-290. (1983).

(17) King, R. B., Encyclopedia of Inorganic Chemistry, vol. 2 (Chichester, New York, 1994).

(18) Nakatsuji, S. et al. Spin disorder on a triangular lattice. Science. 309, 1697-1700. (2005).

(19) Yamashita, S., Yamamoto, T., Nakazawa, Y., Tamura, M. and Kato, R. Gapless spin liquid of an organic triangular compound evidenced by thermodynamic measurements. Nature Comm. 2, 1-6. (2011).

(20) Shores, M. P., Nytko, E. A., Bartlett, B. M., Nocera, D. G. A structurally perfect $S=1 / 2$ Kagome antiferromagnet. J. Am. Chem. Soc. 127, 13462-13463. (2005).

(21) Helton, J.S. et al. Spin dynamics of the spin-1/2 Kagome lattice antiferromagnet $\mathrm{ZnCu}_{3}(\mathrm{OH})_{6} \mathrm{Cl}_{2}$. Phys. Rev. Lett. 98, 107204. (2007).

(22) Okamoto, Y., Nohara, M., Aruga-Katori, H. and Takagi, H. Spin liquid state in the $S=1 / 2$ hyperkagome antiferromagnet $\mathrm{NA}_{4} \mathrm{Ir}_{3} \mathrm{O}_{8}$. Phys. Rev. Lett. 99, 137207. (2007).

(23) Castelnovo, C., Moessner, R., Sondhi, S.L. Magnetic monopoles in spin ice. Nature. 451, 42-45. (2008).

(24) Benbow, E. M., Dalal, N. S., Latturner, S. E. Spin glass behavior of isolated, geometrically frustrated tetrahedra of iron atoms in the intermetallic $\mathrm{La}_{21} \mathrm{Fe}_{8} \mathrm{Sn}_{7} \mathrm{C}_{12}$. J. Am. Chem. Soc. 131, 3349-3354. (2009).

(25) De La Cruz, C. et al. Magnetic order close to superconductivity in the iron based layered $\mathrm{LaO}_{1-\mathrm{x}} \mathrm{F}_{\mathrm{x}} \mathrm{Fe}$ As systems. Nature. 453, 899-902. (2008).

(26) Pene, K., Shannon, N., Shiba, H. Half-magnetization plateau stabilized by structural distortion in the antiferromagnetic Heisenberg model on a pyrochlore lattice. Phys. Rev. Lett. 93,197203. (2004). 
(27) Freedman, D. E. et al. Site specific X-ray anomalous dispersion of the geometrically frustrated Kagome magnet, herbertsmithite, $\mathrm{ZnCu}_{3}(\mathrm{OH})_{6} \mathrm{Cl}_{2}$. J. Am. Chem. Soc. 132, 1618516190. (2010).

(28) Wang. R. F. et al. Artificial 'spin ice' in a geometrically frustrated lattice of nanoscale ferromagnetic islands. Nature. 439, 303-306. (2006).

(29) Torardi, C. C. and McCarley, R. E. Synthesis, crystal structures, and properties of $\mathrm{LiZn}_{2} \mathrm{Mo}_{3} \mathrm{O}_{8}, \mathrm{Zn}_{3} \mathrm{Mo}_{3} \mathrm{O}_{8}$, and $\mathrm{ScZnMo}_{3} \mathrm{O}_{8}$, reduced derivatives containing the $\mathrm{Mo}_{3} \mathrm{O}_{13}$ cluster unit. Inorganic chemistry. 24, 476-481. (1985).

(30) Bridges, C.A., Hansen, T., Wills, A.S., Luke, G.M., Greedan, J.E., Frustrated magnetism and the effects of titanium substitution in the mixed valence oxide $\mathrm{BaV}_{10-\mathrm{x}} \mathrm{Ti}_{\mathrm{x}} \mathrm{O}_{15}$. Phys. Rev. B. 74, 024426. (2006).

(31) Larson, A.C. and Von Dreele, R.B. General Structure Analysis System (GSAS), Los Alamos National Laboratory Report LAUR, 86-748 (1994).

(32) Toby, B. H. EXPGUI, a graphical user interface for GSAS, J. Appl. Cryst. 34, 210213.(2001).

(33) Schmidt, M. W. et al. General atomic and molecular electronic structure system. J. Comput. Chem. 14, 1347-1363. (1993). 


\title{
Supplementary information
}

\section{Possible valence bond condensation in the frustrated cluster magnet $\mathrm{LiZn}_{2} \mathrm{Mo}_{3} \mathrm{O}_{8}$}

\author{
J.P. Sheckelton, J.R. Neilson, D.G. Soltan, and T.M. McQueen
}

Department of Chemistry, Department of Physics and Astronomy, and the Institute for Quantum Matter, The Johns Hopkins University, Baltimore, MD 21218

email: mcqueen@jhu.edu

Index

Figure S1. 12 K powder neutron diffraction and Rietveld refinement.

16

Heat capacity analysis.

Figure S2. Formula scaled $\mathrm{Zn}_{2} \mathrm{Mo}_{3} \mathrm{O}_{8} \mathrm{Cp} \cdot \mathrm{T}^{-1}$ dataset.

Figure S3. Excess Heat capacity plots.

Figure S4. Integrated entropies.

Figure S5. Schottky anomaly analysis.

21

Figure S6. Resistivity measurement from $50 \mathrm{~K}$ to $300 \mathrm{~K}$. 22

Magnetism and spin-orbit coupling considerations.

Figure S7. Magnetic susceptibility analysis details.

Figure S8. Magnetic Kotani behavior analysis.

Table S1. GAMESS input files.

29

30 
a

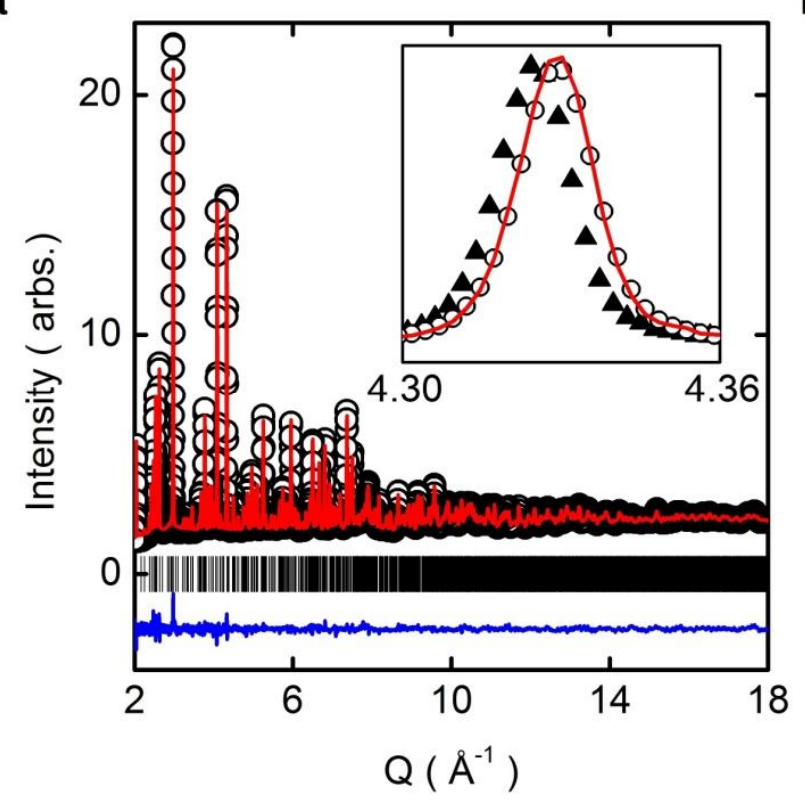

b

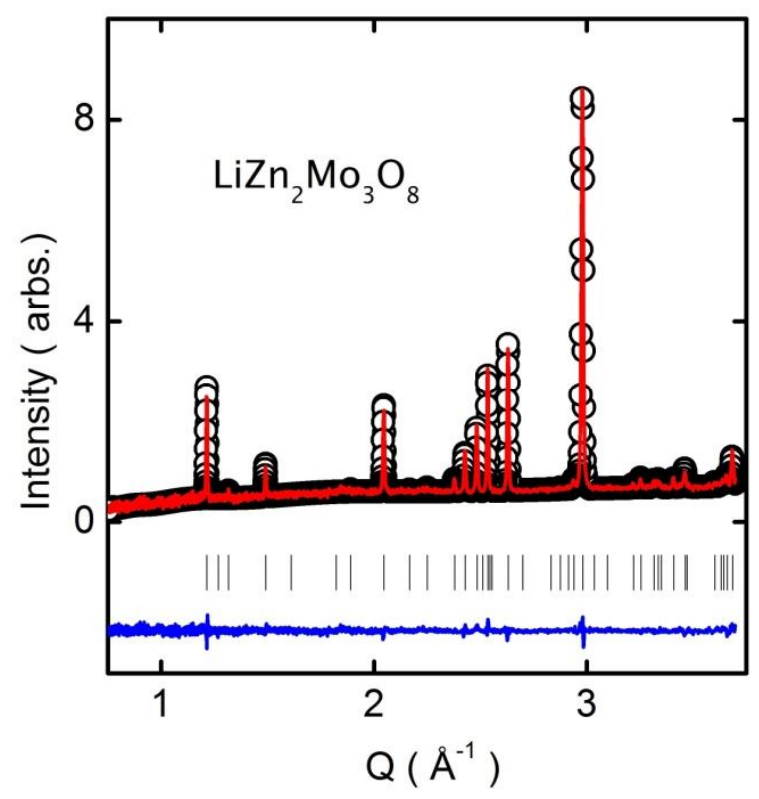

Figure S1 | 12 K TOF neutron powder diffraction data for $\mathrm{LiZn}_{2} \mathrm{Mo}_{3} \mathrm{O}_{8}$ from POWGEN.

a,b. Rietveld refinement of data show that $\mathrm{LiZn}_{2} \mathrm{Mo}_{3} \mathrm{O}_{8}$ remains in the $\mathrm{R} \overline{3} \mathrm{~m}$ spacegroup and maintains its trigonal symmetry in the condensed valence bond state. The inset in a shows the (220) peak at both $T=12 \mathrm{~K}$ (open circles) and at $T=300 \mathrm{~K}$ (triangles), which are sharp and show no indication of a distortion breaking trigonal symmetry. Attempts to fit the patterns to lower symmetry but trigonal models (R3m, R32, R3) did not result in better fits to the data. The patterns show no extra Bragg reflections or diffuse scattering due to magnetic ordering of any kind down to $T=12 \mathrm{~K}$.

As a test, a magnetic phase was added to the refinement to asses if scattering from a magnetic phase would be visible. The magnetic form factor in this material is not known, and there are many possible magnetic orders. Thus to estimate our sensitivity to magnetic order, we used a $120^{\circ}$ magnetic state with the Mo metal form factor. This resulted in an upper limit on the magnetic moment of $0.2(2) \mu_{B}$ per Mo. Together with the absence of an anomaly in the heat capacity, these data suggest no magnetic ordering around $T=96 \mathrm{~K}$. 
Heat capacity analysis.

The magnetic contribution to the heat capacity was extracted by two methods. In the first method, the data for non-magnetic $\mathrm{Zn}_{2} \mathrm{Mo}_{3} \mathrm{O}_{8}$ was scaled to account for the expected change in Debye temperature (compared to $\mathrm{LiZn}_{2} \mathrm{Mo}_{3} \mathrm{O}_{8}$ ) as well as for the change in the number of atoms per formula unit $(=14 / 13)$. Subtraction gives the estimated magnetic heat capacity shown in figure S3(a).

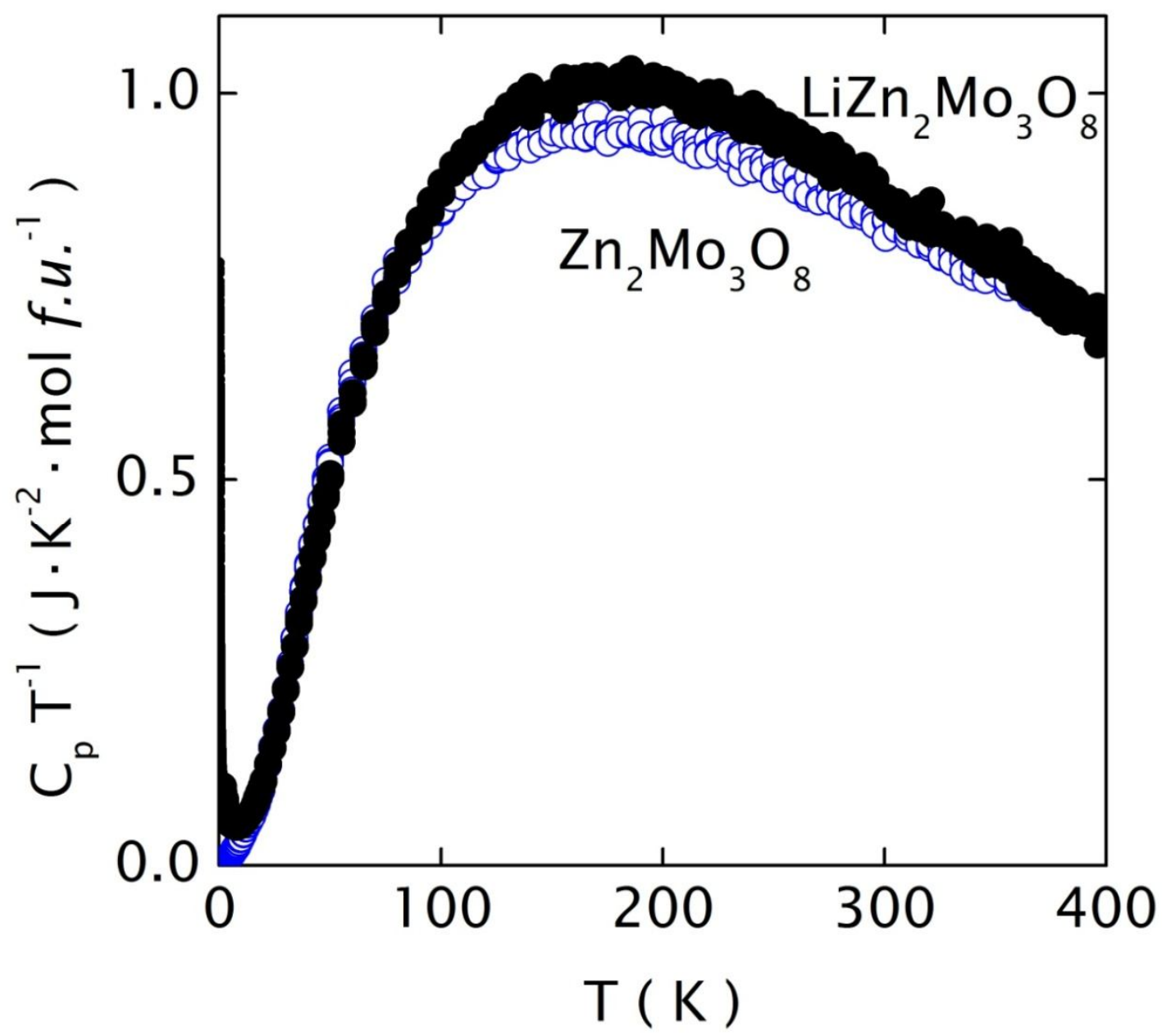

Figure S2 | $\mathrm{LiZn}_{2} \mathrm{Mo}_{3} \mathrm{O}_{8}$ and formula scaled $\mathrm{Zn}_{2} \mathrm{Mo}_{3} \mathrm{O}_{8} \mathrm{Cp} \cdot \mathrm{T}^{-1}$ datasets. 
In the second method, the non-magnetic $\mathrm{Zn}_{2} \mathrm{Mo}_{3} \mathrm{O}_{8}$ data were not scaled for the change in the number of atoms per formula unit (paper Figure 2b). Instead, a smooth fit to the non-magnetic $\mathrm{Zn}_{2} \mathrm{Mo}_{3} \mathrm{O}_{8} \mathrm{C}_{\mathrm{p}} \cdot \mathrm{T}^{-1}$ was directly subtracted, giving the data in figure $\mathrm{S} 3(\mathrm{~b})$, which includes both the magnetic contribution and the extra lattice contribution from the extra lithium atom per formula unit.
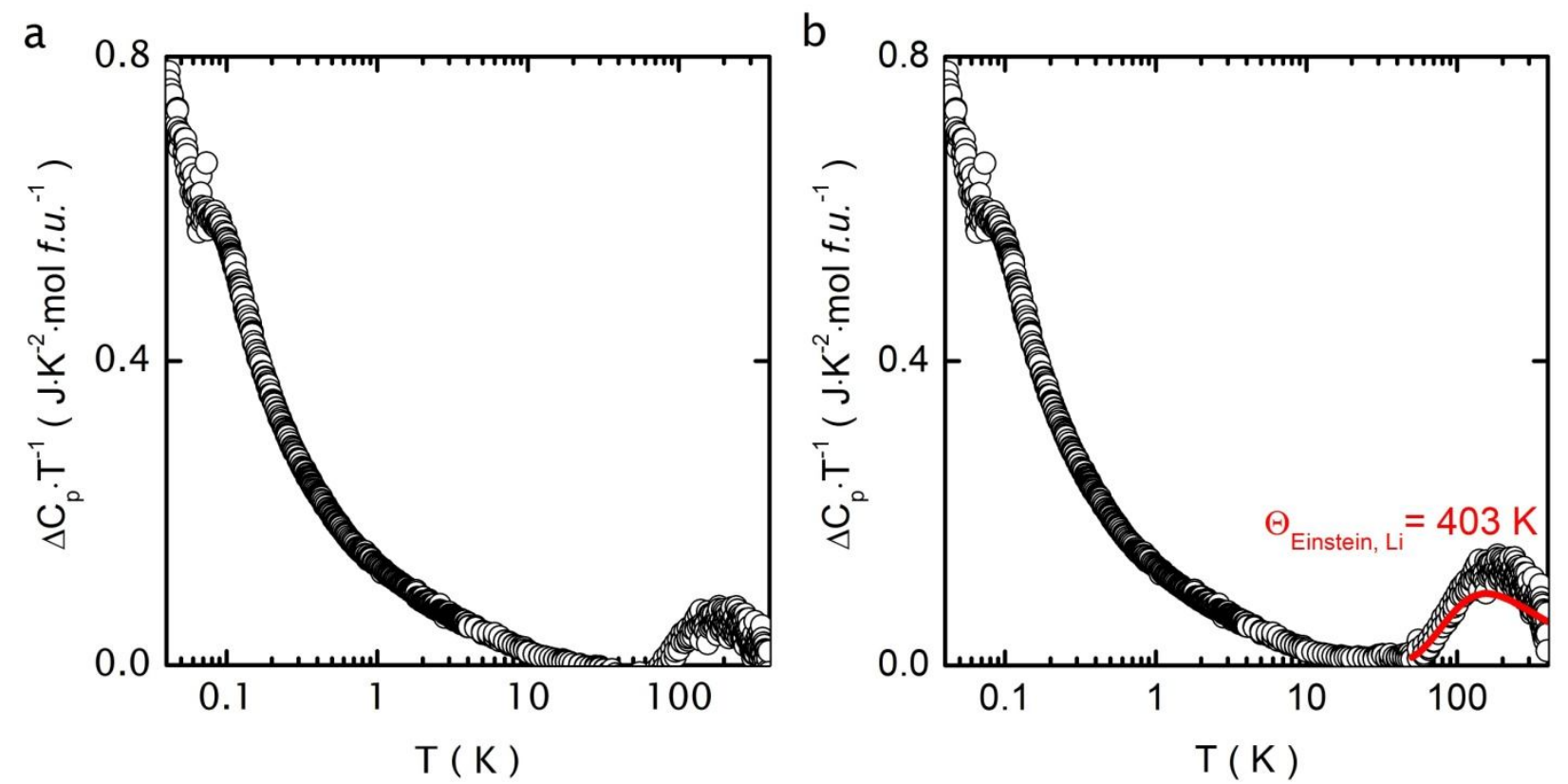

Figure S3 | Estimated excess heat capacity in $\mathrm{LiZn}_{2} \mathrm{Mo}_{3} \mathrm{O}_{8}$ computed by two methods. a, In the first method, the data for non-magnetic $\mathrm{Zn}_{2} \mathrm{Mo}_{3} \mathrm{O}_{8}$ was scaled to account for the expected change in Debye temperature (compared to $\mathrm{LiZn}_{2} \mathrm{Mo}_{3} \mathrm{O}_{8}$ ) as well as for the change in the number of atoms per formula unit, leaving only an estimate of the magnetic entropy. Note the unphysical dip to negative heat capacity around $T=50 \mathrm{~K}$. b, In the second method, the non-magnetic $\mathrm{Zn}_{2} \mathrm{Mo}_{3} \mathrm{O}_{8}$ data were not scaled for the change in the number of atoms per formula unit, leaving contributions from both magnetism and the extra lattice contribution from the extra lithium atom per formula unit. The extra lattice entropy of Li can then be accounted for by fitting to an 
Einstein (or Debye) oscillator mode (fit shown in red).

Both methods give similar insights into the magnetic behavior for $\mathrm{LiZn}_{2} \mathrm{Mo}_{3} \mathrm{O}_{8}$. Method two gives a larger feature at $T \geq 100 \mathrm{~K}$, which must (at least partly) the freezing out of the extra vibrational modes from $\mathrm{Li}$ in $\mathrm{LiZn}_{2} \mathrm{Mo}_{3} \mathrm{O}_{8}$. Figure S3(b) shows a fit of this feature to an Einstein oscillator mode, with an Einstein temperature $\Theta=403 \mathrm{~K}$ (a Debye mode fits equally well), which was subtracted to leave the magnetic contribution. In both cases, the magnetic entropy was then extracted by computing $S(T)=\int_{0}^{T} \frac{C}{T} d T$. A comparison of the two are shown in figure S4.

a

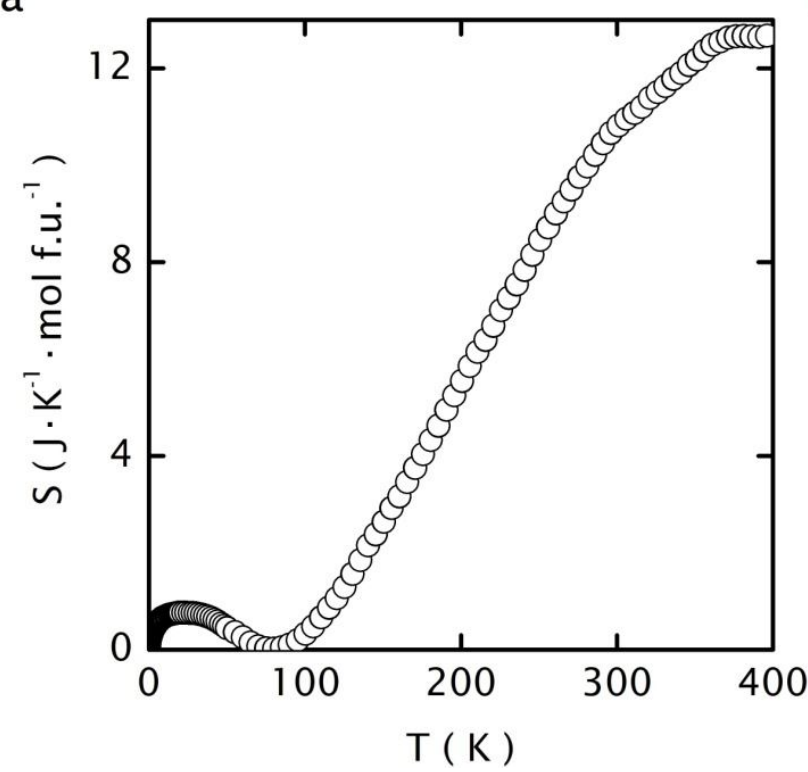

b

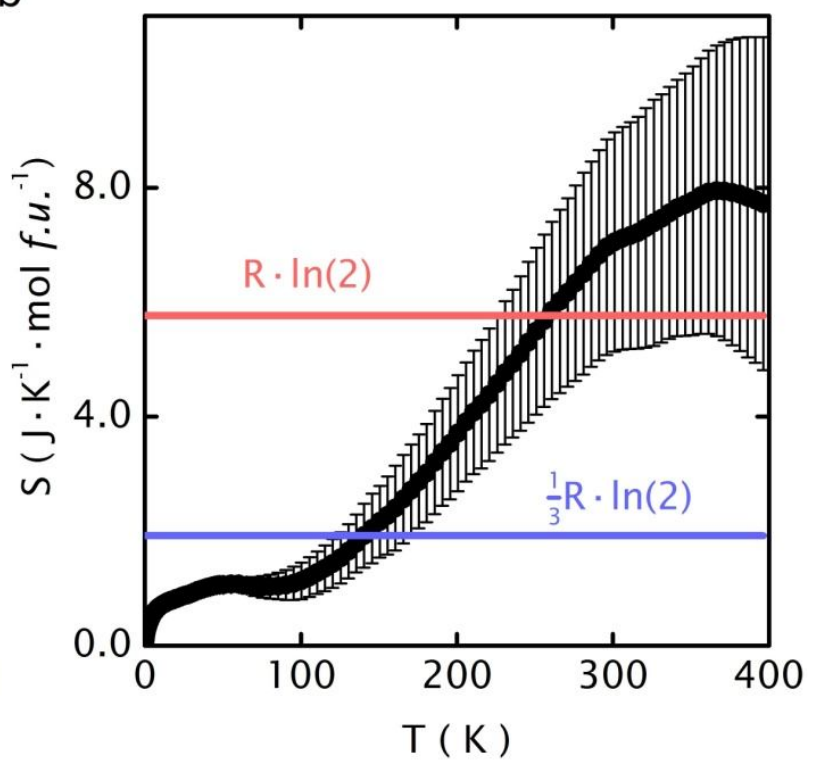

Figure S4 | Estimated magnetic entropy by two methods. a, Integrated $C_{p} \cdot T^{-1}$ data from method one. Although the dip in entropy around $T=50 \mathrm{~K}$ is unphysical, the general result, of two distinct regions of entropy loss - below and above $T \approx 100 \mathrm{~K}$ - is the same as that found by method two. $\mathbf{b}$, Integrated $\mathrm{C}_{\mathrm{p}} \cdot \mathrm{T}^{-1}$ from method two. This data is the same as paper figure $2 \mathrm{c}$, and also shows two distinct regions of entropy loss. 
In both cases, the magnetic entropy contribution contains two discrete regions of entropy gain/loss, below and above $T=100 \mathrm{~K}$. We note that the validity of fitting $\mathrm{C}_{\mathrm{p}}$ to an Einstein (or Debye) oscillator model for the lattice contribution at high temperature (rather than $C_{v}$ ), is justified. We estimate that $C_{v}$ and $C_{p}$ are the same to within approx. $1 \%$ up to $T=400 \mathrm{~K}$ : the relation $C_{P}-C_{v}=A C_{p}^{2} T$ can be used to estimate $C_{v}$, where $A=\beta^{2} V / C_{p}^{2} \kappa_{T}, \beta=V^{-1}\left(\frac{\partial V}{\partial T}\right)_{P}$ is the volume expansion coefficient and $\kappa_{T}=-V^{-1}\left(\frac{\partial V}{\partial P}\right)_{P}$ is the isothermal compressibility ${ }^{34}$. LeBail refinements of the neutron diffraction patterns from $T=300 \mathrm{~K}$ to $T=12 \mathrm{~K}$ were used to estimate $\beta\left(\approx 10^{-5} \mathrm{~K}^{-1}\right)$. The isothermal compressibility was estimated using a literature value for another closest packed oxide $\left(\mathrm{MgO} \kappa_{T}=7.2 \mathrm{~Pa}^{-1}\right.$; virtually all oxides have isothermal compressibilities within one order of magnitude of this value ${ }^{35}$ ).

In both methods, there is a low temperature feature in the magnetic $\mathrm{C}_{\mathrm{p}} \cdot \mathrm{T}^{-1}$ data. Figure $\mathrm{S} 5$ shows an analysis indicating that the feature cannot be adequately explained by simple Schottky anomaly models. Two or three level Schottky anomalies do not account for excess heat capacity in the data, even when other extra terms that might be present are also included. 

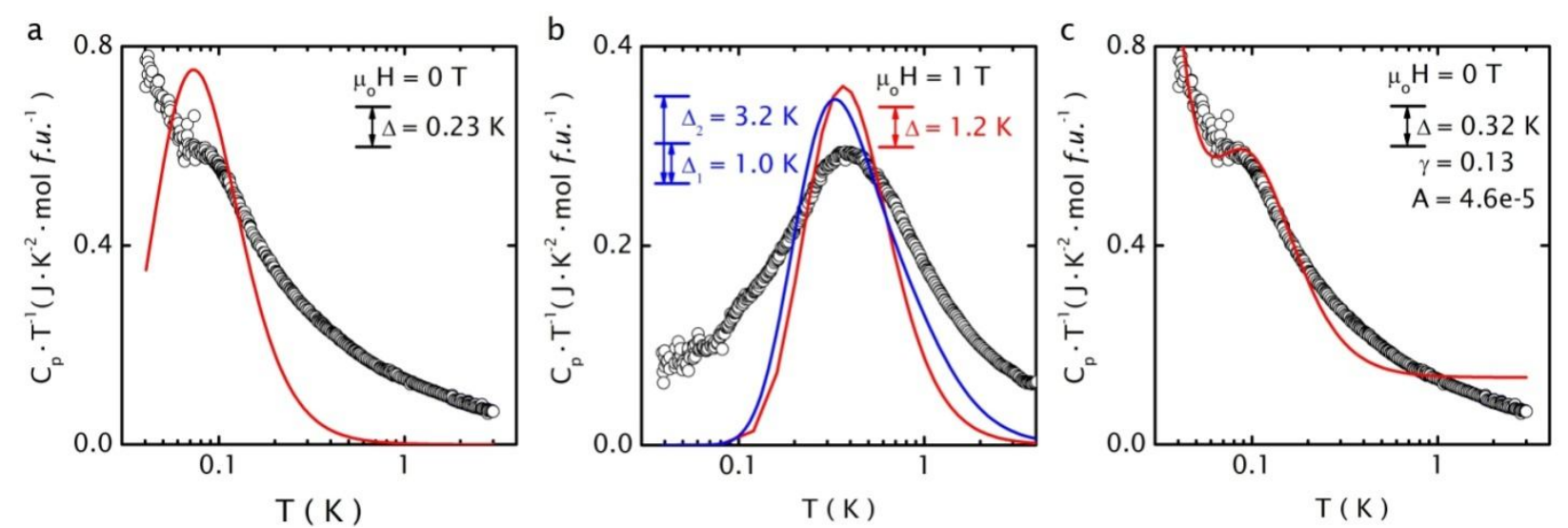

Figure S5 | Low temperature $\mathbf{C}_{\mathbf{p}} \mathbf{T}^{-1}$ Schottky anomaly analysis. a, $\mu_{o} H=0 \mathrm{~T}$ data fit to a two level anomaly and $\mathbf{b}, \mu_{o} H=1 \mathrm{~T}$ fit for both a two (red) and three (blue) level Schottky anomaly. c, A fit to the $\mu_{o} H=0 \mathrm{~T}$ data allowing for a $\gamma \mathrm{T}$ electronic contribution $\left(\gamma\right.$ is in units of $\mathrm{J} \cdot \mathrm{K}^{-2} \cdot \mathrm{mol}$ $f \cdot u .^{-1}$ ) and $\mathrm{AT}^{-2}$ nuclear contribution (A is in units of $\mathrm{J} \cdot \mathrm{K} \cdot \mathrm{mol} f \cdot u{ }^{-1}$ ). Despite allowing for these contributions (a $\gamma \mathrm{T}$ term is unphysical considering the material is an insulator, see figure S6, although such a contribution could arise from spin-liquid behavior) the fit still does not adequately describe the data. In all cases, changing the degeneracy of the anomaly levels $\left(\mathrm{g}_{\mathrm{o}} / \mathrm{g}_{1}\right)$ does not resolve the fit, only changes the width by a small amount. All figures show datasets from both methods of subtracting the lattice contribution of $\mathrm{Zn}_{2} \mathrm{Mo}_{3} \mathrm{O}_{8}$, but at these temperatures, the difference is negligible. 

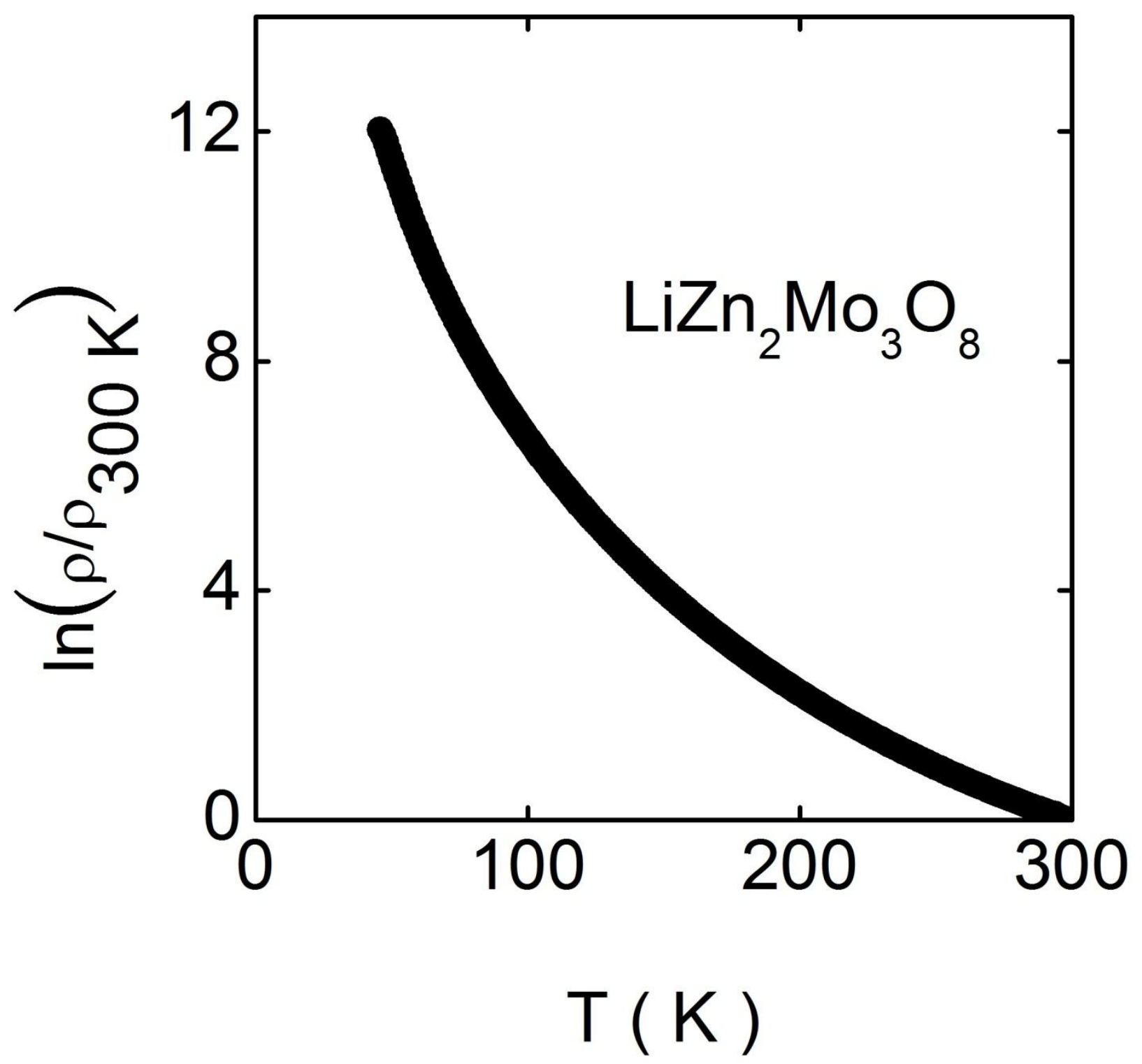

Figure S6 | $\mathrm{LiZn}_{2} \mathrm{Mo}_{3} \mathrm{O}_{8}$ resistivity. Resistivity as a function of temperature data measured on a sintered pellet of $\mathrm{LiZn}_{2} \mathrm{Mo}_{3} \mathrm{O}_{8}$. Measurements taken down to $T=50 \mathrm{~K}$ (where the voltmeter saturated) show that $\mathrm{LiZn}_{2} \mathrm{Mo}_{3} \mathrm{O}_{8}$ is an electrical insulator and shows no obvious signs of electronic transitions. This is as expected for the formation of a condensed valence bond state (which occurs as $\mathrm{T}=96 \mathrm{~K}$ ). The high temperature data was fit to a model $\rho=\rho_{o} e^{E_{g} / 2 K_{B} T}$ giving an estimated band gap of $E_{g}=0.12 \mathrm{eV}$. 


\section{Magnetism and spin-orbit coupling considerations.}

In $4 \mathrm{~d}$ and 5d systems, spin orbit (SOC) contributions to magnetism can be significant. The spinorbit coupling constant for Mo is $\xi=0.068 \mathrm{eV}$, similar to the value for $\mathrm{Cu}, \xi=0.100 \mathrm{eV}^{37}$. Furthermore, the crystal field splitting of adjacent states in $\mathrm{Mo}_{3} \mathrm{O}_{13}$ cluster is $\sim 1.2 \mathrm{eV}$, on the same scale as in $\mathrm{Cu}^{2+}$ compounds $(\sim 2.4 \mathrm{eV})$. Thus the effects of SOC are expected to be similar, within a factor of 2. To more quantitatively assess the expected effect of spin orbit coupling on the observed magnetism, we calculated the expected deviation in the magnetic g-factor arising from a partial unquenched orbital contribution (from SOC) in a perturbative manner. To second $\operatorname{order}^{36}$, the observed g-value, $\mathrm{g}_{\mathrm{m}}$, is given by $g_{m}=g_{e}\left(1-A \frac{\xi}{\Delta E}\right)$ where $g_{e}$ is the ideal value (= 2.0), $\xi$ is the spin-orbit coupling constant, and $\Delta E$ is the energy gap between electronic states from crystal field effects, and $A$ is a constant dependant on the exact nature of the ground and low-lying electronic states. The values of $\xi$ are taken from the literature ${ }^{37}$ and values for $A$ are typically in the range of 2-4. Performing calculations using the extremes of A, we see that $g_{m}$ ranges from 1.55 to 1.77 , consistent with a g-factor value extracted from the high-temperature Curie constant of $0.24 \mathrm{emu} \cdot \mathrm{K} \cdot \mathrm{Oe}^{-1} \cdot \operatorname{mol} f \cdot u^{-1}$ found in our data $\left(g_{m} \approx 1.6\right)$. Performing this same type of calculation on $\mathrm{Cu}$ yields a g-factor range of 2.18 to 2.37 , consistent with experiment $\left(g_{m} \approx 2.2\right)$. This shows that spin-orbit coupling can be an explanation as to the reduced value of the Curie constant (and hence $\mu_{e f f}$ ) observed in our inverse susceptibility data.

However, while spin-orbit coupling can itself give rise to interesting magnetic phases ${ }^{38}$, an analysis carried out in the same way as Kotani show that, in this case, it does not explain the observed trends in the magnetic data, notably the transition around $T=96 \mathrm{~K}$. 


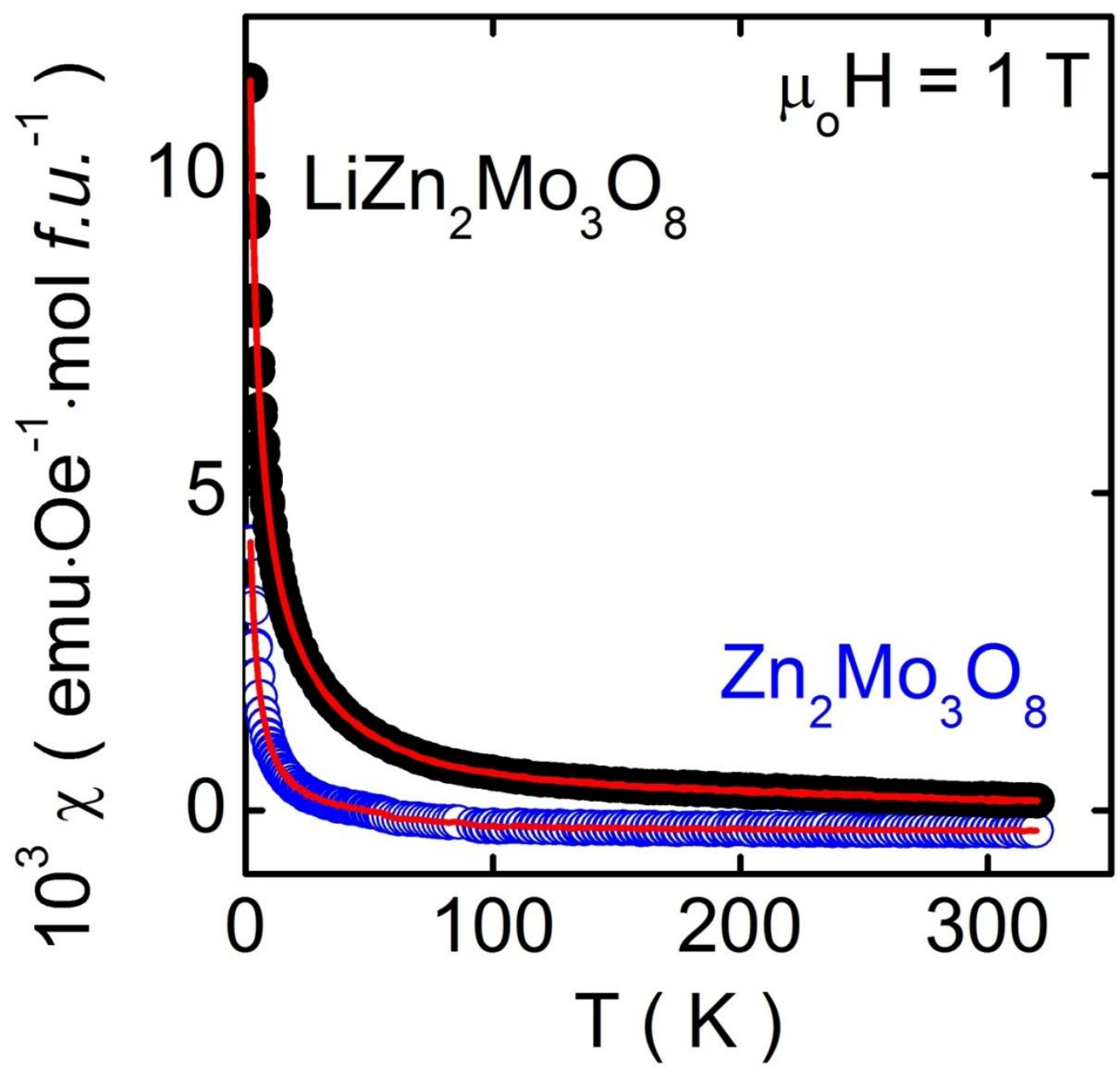

Figure $\mathrm{S} 7 \mid \mathrm{LiZn}_{2} \mathrm{Mo}_{3} \mathrm{O}_{8}$ and $\mathrm{Zn}_{2} \mathrm{Mo}_{3} \mathrm{O}_{8}$ magnetic susceptibility. Magnetic susceptibility data as a function of temperature for $\mathrm{LiZn}_{2} \mathrm{Mo}_{3} \mathrm{O}_{8}$ and $\mathrm{Zn}_{2} \mathrm{Mo}_{3} \mathrm{O}_{8}$. DC magnetization was measured on equimolar amounts $(0.112 \mathrm{mmol})$ of each compound using the same sample container. This allowed for direct subtraction of the two datasets to determine the intrinsic response of the unpaired electrons in $\mathrm{LiZn}_{2} \mathrm{Mo}_{3} \mathrm{O}_{8} . \mathrm{M}(\mathrm{H})$ curves were linear up to $\mu_{o} H=1 \mathrm{~T}$, the applied field used for the measurements, and thus the susceptibility was calculated assuming $\chi=\frac{M}{H}$. The small Curie tail on $\mathrm{Zn}_{2} \mathrm{Mo}_{3} \mathrm{O}_{8}$ corresponds to $4.4 \%$ of paramagnetic impurity spins, mostly from the sample holder. 

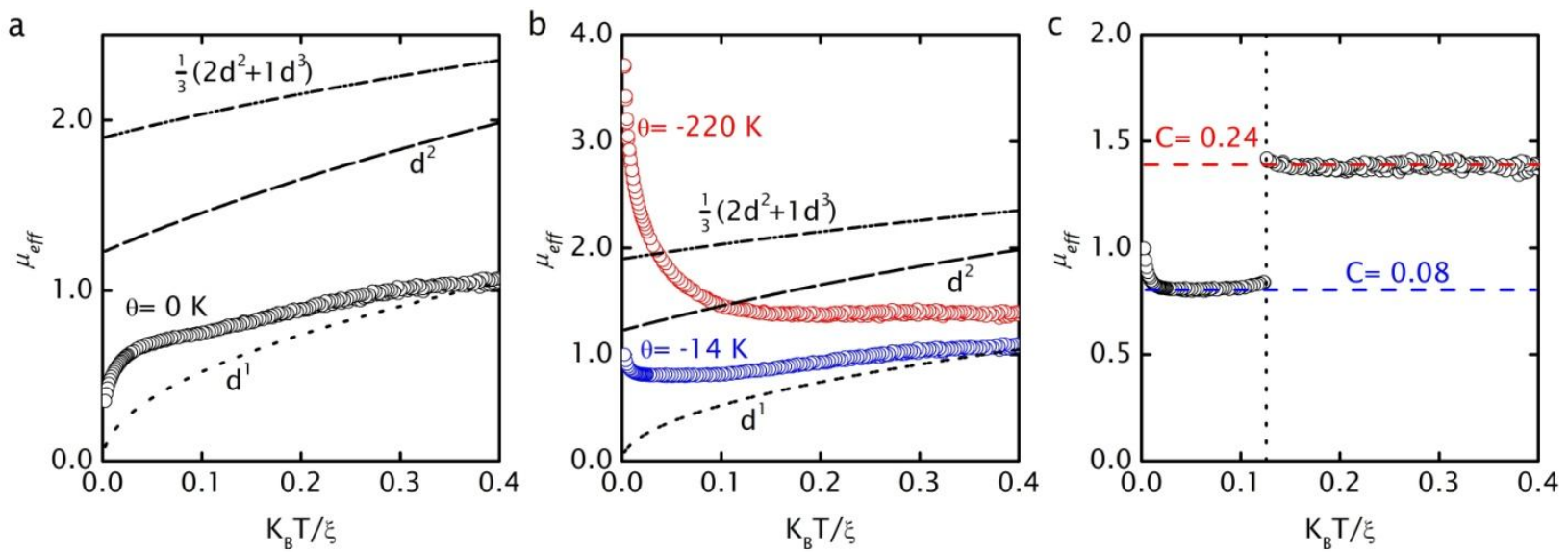

Figure S8 | $\mathrm{LiZn}_{2} \mathrm{Mo}_{3} \mathrm{O}_{8}$ temperature dependence of effective magnetic moments.

Calculations of effective magnetic moments on $\mathrm{LiZn}_{2} \mathrm{Mo}_{3} \mathrm{O}_{8}$ as a function of thermal energy $\left(\mathrm{k}_{\mathrm{B}} \mathrm{T}\right)$ divided by the spin orbit coupling constant $(\xi=0.068 \mathrm{eV})$ for molybdenum. The formula $\mu_{e f f}=\sqrt{\frac{3 k_{B}}{N_{A} \mu_{B}^{2}}\left(\chi-\chi_{o}\right)(T-\theta)}$ is used to calculate the effective magnetic moment where $\chi$ is the measured magnetic susceptibility, $\chi_{o}$ is the temperature independent contribution to the susceptibility (in this case $=0$ because of the subtraction of $\mathrm{Zn}_{2} \mathrm{Mo}_{3} \mathrm{O}_{8}$ and sample holder susceptibility), $T$ is temperature and $\theta$ is the Weiss temperature - indicative of the magnitude and sign of interactions between magnetic moments. $\mu_{e f f}$ is in units of $\mu_{B}$ per $f . u$. The plots show the calculated magnetic moment for a, no interactions $(\theta=0 \mathrm{~K})$, $\mathbf{b}$, weak $(\theta=-14 \mathrm{~K})$ or strong $(\theta=-$ 220K) antiferromagnetic interactions uniform over all temperatures, and $\mathbf{c}$, a change from strong to weak antiferromagnetic interactions at $T \approx 96 \mathrm{~K}$. In $\mathbf{a}$ and $\mathbf{b}$, the expected Kotani behaviors for $\mathrm{d}^{2}$ ions, $\mathrm{d}^{1}$ ions, and a scaled (by one-third) combination of $2 \mathrm{~d}^{2}$ and $1 \mathrm{~d}^{3}$ ions are shown. The $\mathrm{d}^{1}$ case is similar to our proposed spin-1/2 degree of freedom magnetic molybdenum clusters, and the linear combination of $\mathrm{d}^{2}$ and $\mathrm{d}^{3}$ case is what one would expect if each cluster were comprised of two $\mathrm{Mo}^{4+}$ and one $\mathrm{Mo}^{3+}$ distinct ions. The observed data are not consistent with any of these 
Kotani behaviors, as can be seen; the shape of the data is inconsistent with a change in unquenched orbital contribution with temperature (which is the hallmark of SOC Kotani behavior). In c, the extracted Weiss temperatures from fits to the inverse susceptibility data are used for the temperature ranges from which they are extracted. In this case the appearance of two flat regions above and below this transition indicates a loss of a large contribution to the effective magnetic moment around this temperature. This supports the argument that $\mathrm{LiZn}_{2} \mathrm{Mo}_{3} \mathrm{O}_{8}$ behaves as an isolated $\mathrm{S}=1 / 2$ system with a partial unquenched orbital contribution that is temperatureindependent (just like $\mathrm{Cu}^{2+}$ compounds). 


\section{Table S1 | GAMESS input files:}

GAMESS input files for calculations on a $\mathrm{Mo}_{3} \mathrm{O}_{4}(\mathrm{OH})_{3}\left(\mathrm{H}_{2} \mathrm{O}\right)_{6}$ cluster using unrestricted spin density functional theory with the PBE0 hybrid functional and Popel's N-21 split valence basis set with 3 gaussians for Mo, the 6-31G(d) basis for $\mathrm{O}$ and the 6-31G basis for $\mathrm{H}^{39,40}$. $\mathrm{Mo}_{3} \mathrm{O}_{4}(\mathrm{OH})_{3}\left(\mathrm{H}_{2} \mathrm{O}\right)_{6}$ was chosen because it is a neutral molecule, has the same electron count as the $\mathrm{Mo}_{3} \mathrm{O}_{13}$ clusters in $\mathrm{LiZn}_{2} \mathrm{Mo}_{3} \mathrm{O}_{8}$, and maintains the $\mathrm{C}_{3 \mathrm{v}}$ symmetry. Input file (a) uses Huzinaga's minimal basis set to provide a suitable initial guess for the electron densities, which is then used as input for the full calculation, using input file (b). Changes to the hybrid functional or basis sets used in (b) results in only minor changes to the calculated frontier orbitals. Spinorbit coupling effects are not included in these calculations.

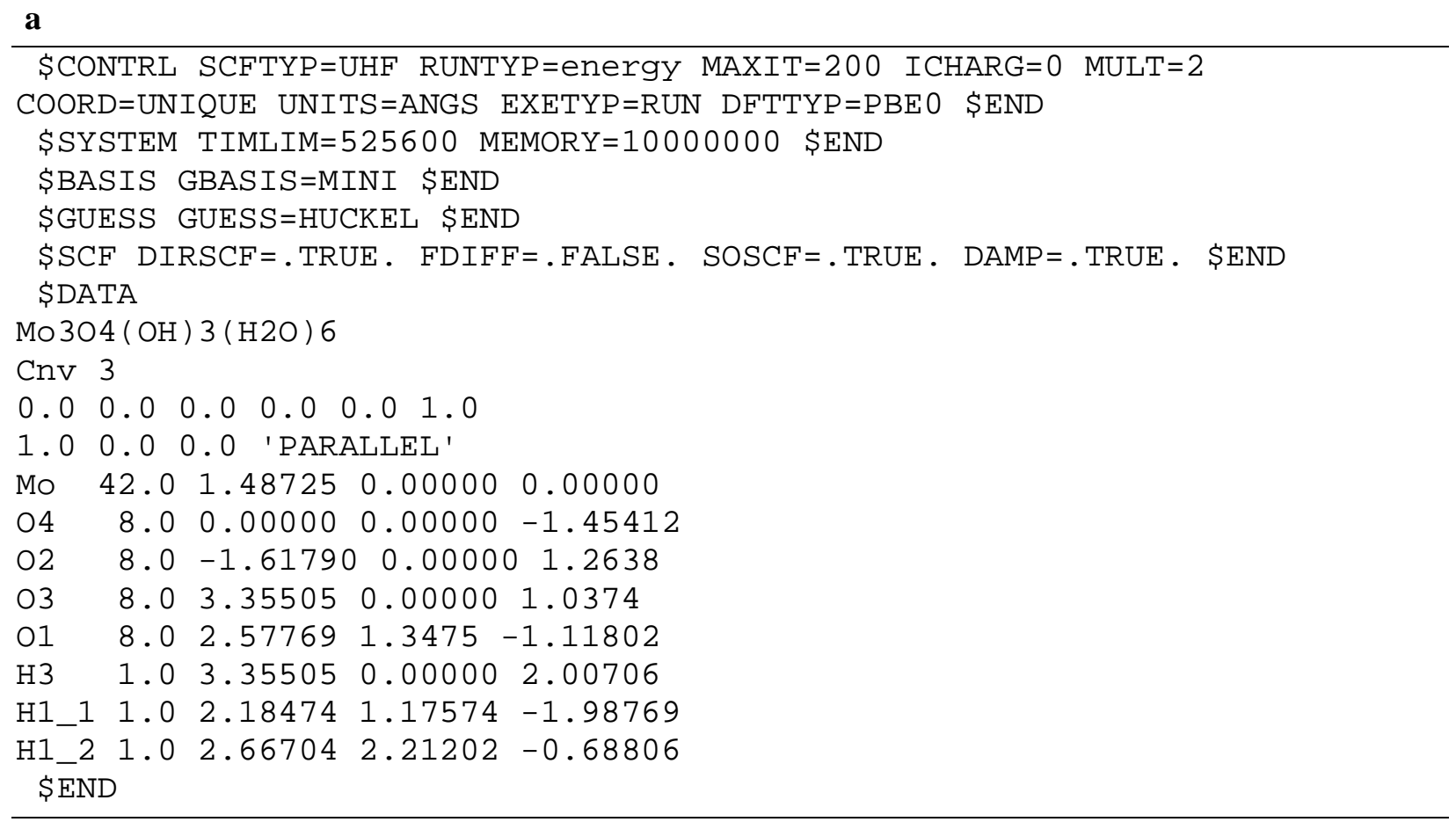




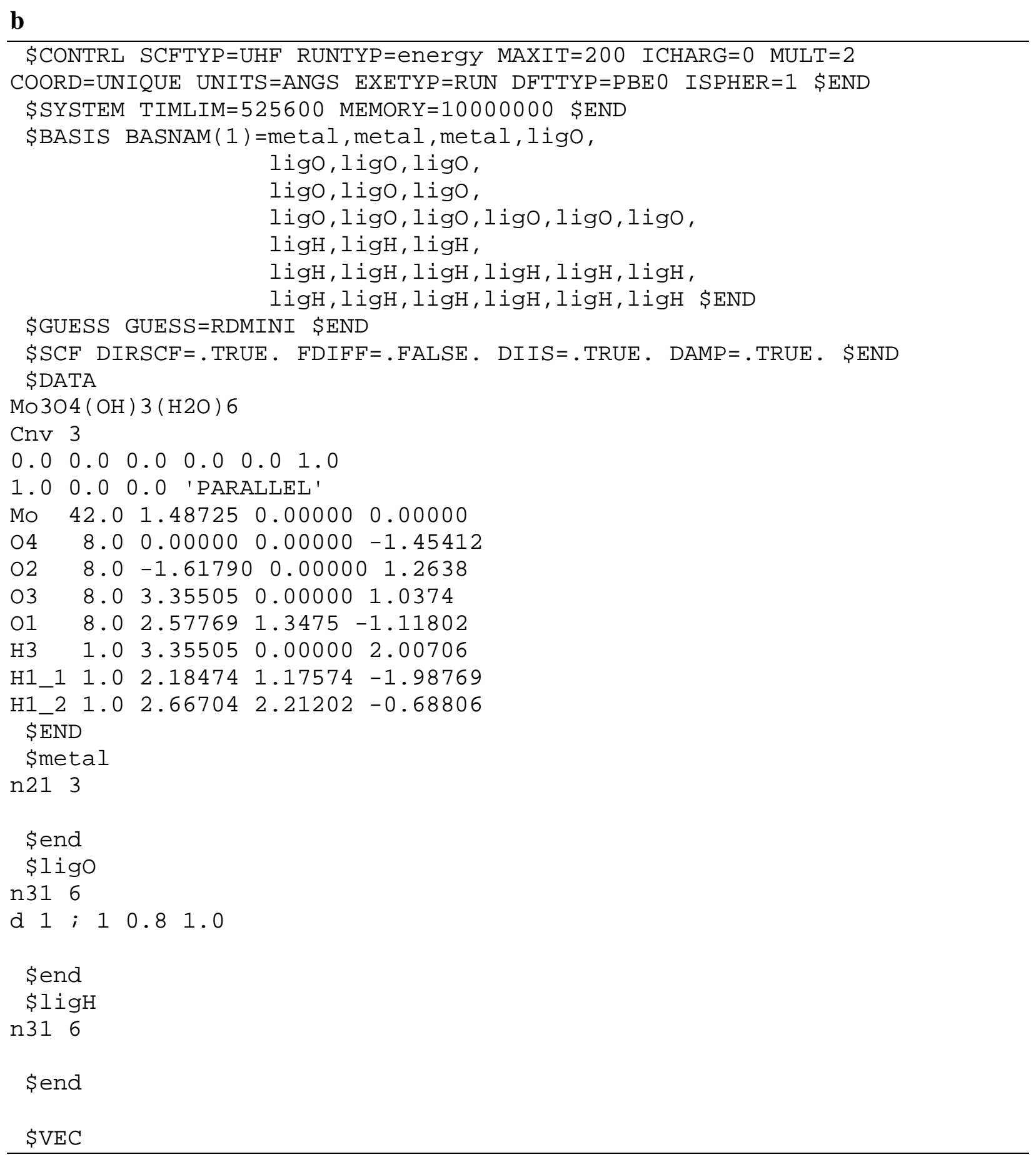


Table S2 | Crystallographic information

\begin{tabular}{|c|c|}
\hline \multicolumn{2}{|c|}{$\mathrm{LiZn}_{2} \mathrm{Mo}_{3} \mathrm{O}_{8}$ refinement } \\
\hline Chemical formula sum & Li1.2(1) Zn1.8(1) Mo3 O8 \\
\hline Space group & $R \overline{3} m$ \\
\hline $\mathrm{a}(\AA)$ & $5.7956(3)$ \\
\hline $\mathrm{b}(\AA)$ & $5.7956(3)$ \\
\hline$c(\AA)$ & $31.039(3)$ \\
\hline $\mathrm{Z}$ & 6 \\
\hline$w_{\mathrm{p}}$ & 0.0303 \\
\hline $\mathrm{R}_{\mathrm{p}}$ & 0.0445 \\
\hline $\mathrm{R}\left(\mathrm{F}^{2}\right)$ & 0.0846 \\
\hline$\chi^{2}$ & 8.356 \\
\hline LeBail $\chi^{2}$ & 2.705 \\
\hline
\end{tabular}

\begin{tabular}{|c|c|c|c|c|c|c|}
\hline \multicolumn{7}{|c|}{$\mathrm{LiZn}_{2} \mathrm{Mo}_{3} \mathrm{O}_{8}$ structural parameters } \\
\hline atom & $\mathbf{x}$ & $\mathbf{y}$ & $\mathbf{z}$ & Wyck. pos. & Occ & $\mathbf{U}_{\text {iso }}$ \\
\hline Mo & $0.18573(7)$ & $0.81428(7)$ & $0.08401(4)$ & $18 \mathrm{~h}$ & 1.000 & $0.0033(1)$ \\
\hline $\mathrm{O} 1$ & $0.84500(9)$ & $0.15498(9)$ & $0.04766(3)$ & $18 \mathrm{~h}$ & 1.000 & $0.0012(1)$ \\
\hline $\mathrm{O} 2$ & $0.49217(10)$ & $0.50783(10)$ & $0.12404(3)$ & $18 \mathrm{~h}$ & 1.000 & $0.0060(1)$ \\
\hline $\mathrm{O} 3$ & 0.0000 & 0.0000 & $0.11820(7)$ & $6 c$ & 1.000 & $0.0052(2)$ \\
\hline $\mathrm{O} 4$ & 0.0000 & 0.0000 & $0.37178(6)$ & $6 c$ & 1.000 & $0.0029(2)$ \\
\hline Zn1 & 0.3333 & 0.6667 & $-0.64148(6)$ & $6 c$ & $0.901(4)$ & $0.0014(1)$ \\
\hline $\mathrm{Zn} 2$ & 0.0000 & 0.0000 & $0.18132(9)$ & $6 c$ & $0.716(4)$ & $0.0014(1)$ \\
\hline Li1 & 0.3333 & 0.6667 & $-0.64148(6)$ & $6 c$ & $0.099(4)$ & $0.0014(1)$ \\
\hline $\mathrm{Zn} 3$ & 0.0000 & 0.0000 & 0.0000 & $3 a$ & $0.226(7)$ & $0.0014(1)$ \\
\hline $\mathrm{Li} 2$ & 0.0000 & 0.0000 & $0.18132(9)$ & $6 c$ & $0.284(4)$ & $0.0014(1)$ \\
\hline $\mathrm{Zn} 4$ & 0.0000 & 0.0000 & 0.5000 & $3 a$ & $0.143(6)$ & $0.0014(1)$ \\
\hline Li3 & 0.0000 & 0.0000 & 0.5000 & $3 a$ & $0.774(7)$ & $0.0014(1)$ \\
\hline $\mathrm{Li} 4$ & 0.0000 & 0.0000 & 0.5000 & $3 a$ & $0.857(6)$ & $0.0014(1)$ \\
\hline
\end{tabular}




\section{Supplementary References}

(34) Tari, A. The Specific Heat of Matter at Low Temperatures, Ch. 1 (Imperial College press, London, 2003)

(35) Washburn, E.W. (1926 - 1930;2003). International Critical Tables of Numerical Data, Physics, Chemistry and Technology (1st Electronic Edition). Knovel. http://www.knovel.com/web/portal/browse/display?_EXT_KNOVEL_DISPLAY_bookid=7 $35 \&$ VerticalID $=0$

(36) Slichter, C.P., Principles of Magnetic Resonance, $3^{\text {rd }}$ ed., Ch. 11 (Springer-Verlag, New York, 1990)

(37) Vijayakumar, M. and Gopinathan, M.S. Spin-orbit coupling constants of transition metal atoms and ions in density functional theory. J. Molecular Structure (Theochem). 361, 15-19 (1996).

(38) Chen, G., Pereira, R., Balents, L., Exotic phases induced by strong spin-orbit coupling in ordered double perovskites. Phys. Rev. B. 82, 174440. (2010).

(39) Binkley, J.S., Popel, J.A., Hehre, W.J. Self-consistent molecular orbital methods. 21. Small split-valence basis sets for first-row elements. J. Am. Chem. Soc. 102, 939-947 (1980).

(40) Dobbs, K.D., Hehre, W.J. Molecular orbital theory of the properties of inorganic and organometallic compounds 5. Extended basis sets for first-row transition metals. J. Comput. Chem. 8, 880-893. (1987). 